\title{
Study on Influence of Crack on the Blade Status Using FBGs
}

\author{
Gang XU ${ }^{1,3^{*}}$, Lei LIANG ${ }^{1}$, Jianzhi LI ${ }^{2}$, Huaping MEI ${ }^{3}$, Hongli $\mathrm{LI}^{3}$, and Yijun LIU' \\ ${ }^{1}$ National Engineering Laboratory of Fiber Optic Sensing Technology, Wuhan University of Technology, Wuhan, \\ 430070, China \\ ${ }^{2}$ The Key Laboratory for Health Monitoring and Control of Large Structures, Shijiazhuang, 050043, China \\ ${ }^{3}$ School of Mechanical Engineering, Hubei Engineering University, Xiaogan, 432000, China \\ *Corresponding author: Gang XUＥ-mail: xugang524@163.com
}

\begin{abstract}
The status detection for rotating parts is difficult since the sensor is influenced by the rotation in the inflammable, explosive, and strong magnetic environment. Based on the fiber Bragg grating sensing technology, this paper studies the influence of the natural frequency and deformation of a rotor blade affected by the size of crack in the blade. Test results show that the speed of the equipment and blade excited vibration frequency are two main factors or deformation and vibration frequency of the blade. With an increase in the crack depth, the blade deformation is increased while the stimulated natural frequency of the blade is decreased; at a low rotational speed, the deformation is mainly caused by the rotating speed of the blade. On the contrary, the vibration blade itself contributes to the deformation at a high speed. During the process of full speed rotation, the influence of the rotational speed on the blade deformation almost remains the same, and the influence of the natural vibration on blade deformation is increased with an increase in the rotational speed.
\end{abstract}

Keywords: Rotor blade; fiber Bragg grating; crack depth; rotational speed; blade frequency; blade deformation

Citation: Gang XU, Lei LIANG, Jianzhi LI, Huaping MEI, Hongli LI, and Yijun LIU, "Study on Influence of Crack on the Blade Status Using FBGs," Photonic Sensors, 2018, 8(1): 63-69.

\section{Introduction}

Referring to any equipment of the condition detection, the use of the correct detection methods, the right equipment, the access to accurate test data, and a reasonable analysis of the data are the basic guarantee to detect the operation of the equipment and identify the cause of the faults. With the development of rotating machinery in the large-scale and precise directions, the requirement of detection of equipment operating status is also higher, which is also important in terms of safety and economy [1-2]. There is no doubt that rotating machinery has always been the most common equipment in a variety of workplaces, and the detection methods are varied. But the detection of rotating parts (blades, shafts, etc) has been one of the difficulties. Since the rotating member is constantly rotating, it is easy to cause the transmission line to be wound, and it is difficult to mount the sensor directly on the detecting member. Usually, we use indirect measurement methods, such as the use of acceleration sensors to measure the vibration of the bearing parts and reflect the condition of the shaft and blades indirectly [3-5]. In a lot of flammable, explosive, and strong magnetic fields, there are

Received: 25 June 2017 / Revised: 30 October 2017

(C) The Author(s) 2017. This article is published with open access at Springerlink.com

DOI: $10.1007 / \mathrm{s} 13320-017-0451-\mathrm{z}$

Article type: Regular 
higher requirements of the sensor, which is even more difficult to detect the safe operation of equipment [6].

In this paper, the authors use the fiber grating sensing technology to measure rotating parts of mechanical equipment from different inspection angles. The fiber grating sensing technology has the advantages of anti-flammable and anti-magnetic field interference, and there are many ways to achieve a direct measurement of rotating parts such as blades, to enhance the accuracy of the measurement.

\section{Experimental principle}

The dynamic homogeneous and strained central wavelength of the reflection spectrum of the fiber Bragg grating (FBG) is symmetrical, and its relationship with strain and the change in temperature is [7]

$$
\begin{aligned}
\lambda(t)= & \lambda+\lambda\left(1-p_{e}\right) \varepsilon\left(z_{\text {mid }}, t\right)+ \\
& \lambda\left[\zeta_{s}+\left(1-p_{e}\right)\left(\alpha_{s}-\alpha_{f}\right)\right] \Delta T
\end{aligned}
$$

where $\varepsilon\left(z_{\text {mid }}, t\right)$ is the time varying strain of the midpoint of the FBG, $\Delta T$ is the change in temperature, $p_{e}=0.22$ is the elasto-optical coefficient of the fiber, $\zeta_{s}=6.45 \times 10^{-6} /{ }^{\circ} \mathrm{C}$ is the thermo-optic coefficient of the fiber, and $\alpha_{s}=11.6 \times 10^{-6} /{ }^{\circ} \mathrm{C}$ and $\alpha_{f}=0.55 \times 10^{-6} /{ }^{\circ} \mathrm{C}$ are the linear expansion coefficients of the fiber and the tested material, respectively.

In the process of high-speed operation, the blade is subjected to the strong centrifugal force, and the centrifugal force on the quality of any micro-section of the blade has the tendency to bring the blade back to the equilibrium position, which is equivalent to the elastic restoring force of the blade. When the blade rotates away from the equilibrium position, its centrifugal force always has a tendency to prevent the blade from bending, which is equivalent to an increase in the stiffness of the blade, so that the moving frequency of the blade is higher than the static frequency. The general formula for calculating the frequency is [8]

$$
f_{d}=\sqrt{f_{A}^{2}+B \cdot f_{r}^{2}}
$$

where $f_{A}$ is the static natural frequency of the speed-frequency, $f_{r}$ is the rotate frequency, and $B$ is dynamic frequency coefficient of the blade, which is always determined by the empirical formula in the practical engineering application.

\section{Experimental methods and equipments}

The experimental equipment is shown in Fig. 1. All the systems are fixed on the cast iron platform. The rotating part is driven by a $2.2 \mathrm{~kW}$ motor with a maximum speed of $2800 \mathrm{rpm}$, the speed is accurately controlled by the inverter, and the blades are fastened to the hub by screws. In order to avoid winding of the fiber during rotation, the signal of the fiber grating is transmitted by the fiber optic rotary joint, which is mounted on the other side of the hub and requires coaxial with the hub. The fiber grating wavelength demodulation system adopts a demodulation system developed by the National Engineering Laboratory of Optical Fiber Sensing of Wuhan University of Technology. The sampling frequency is $4000 \mathrm{~Hz}$, and the measurement accuracy is $1 \mathrm{pm}$. The high speed and high precision demodulation of the fiber grating is realized to meet the experimental requirements.

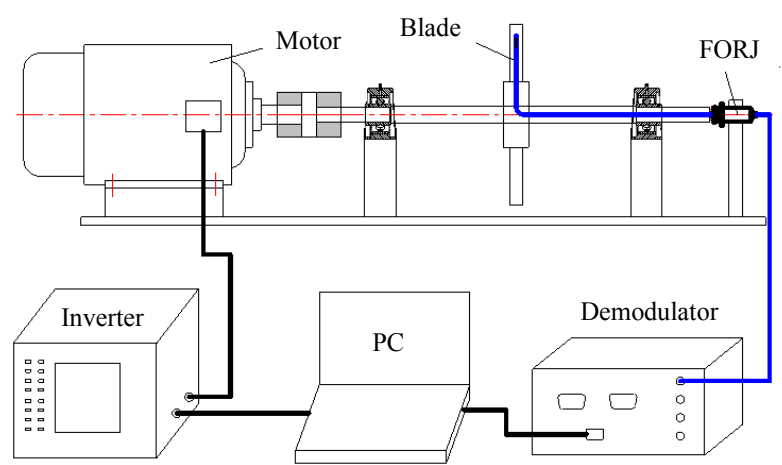

Fig. 1 Diagram of experimental equipment.

The blade morphology is as shown in Fig.2. Its effective length is $80 \mathrm{~mm}$. The arc transition angles are used to lower the stress concentration coefficient of blade roots. The width is $20 \mathrm{~mm}$, and the thickness is $2 \mathrm{~mm}$. The crack is processed by the femtosecond laser, whose width is about $30 \mu \mathrm{m}$, 
whose length runs through the entire blade width, and the depth of the crack can be controlled according to the experimental requirement. The distance of the crack from the end of the blade is $70 \mathrm{~mm}$. The grating is attached to the back of the crack by $353 \mathrm{ND}$ glue.

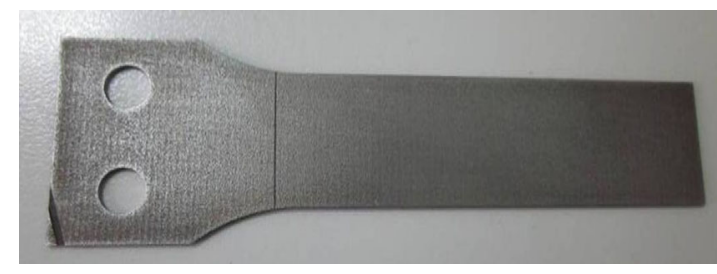

Fig. 2 Blade morphology.

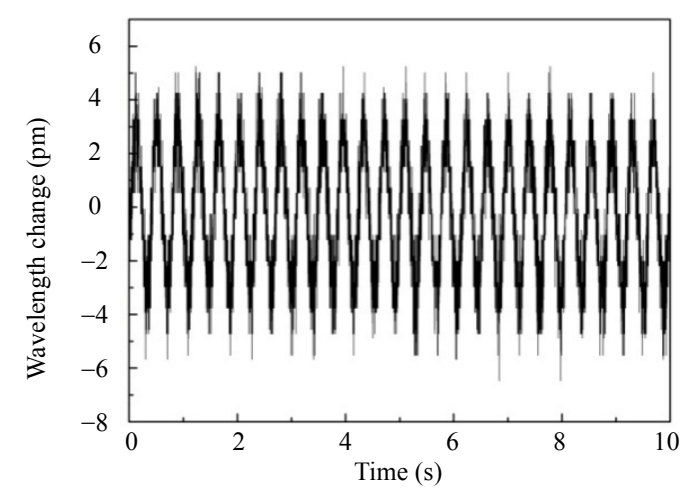

(a)

\section{Experimental results and analysis}

The following analysis of the test results is carried out with the crack depth $h=0.5 \mathrm{~mm}$. Figure 3 shows the time domain diagram and the frequency domain of the Fourier transform in the case of a blade speed of $156 \mathrm{rpm}$. As can be seen from the figure, the blade deformation frequency mainly includes the rotational frequency and the blade natural frequency. The main reason for the deformation of the blade is the speed of the mechanical system itself. The influence of the leaf vibration on the deformation of the blade is small.

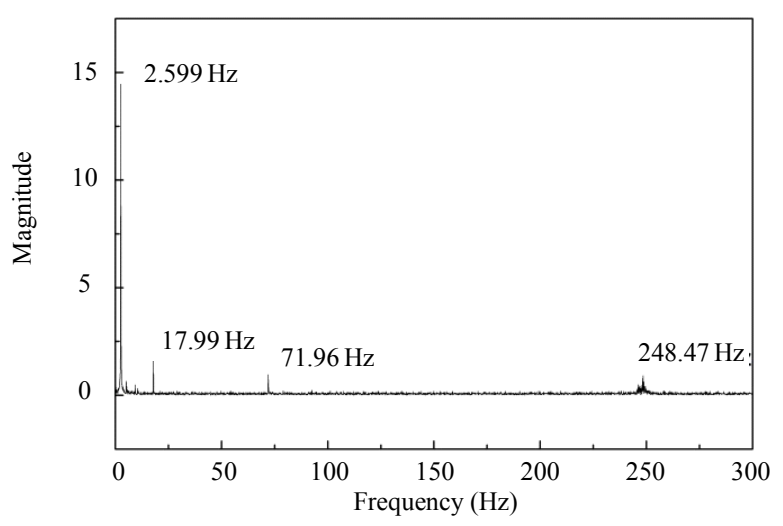

(b)

Fig. 3 Leaf time domain and frequency domain: (a) time domain and (b) signal frequency spectrum.

In order to analyze the influences of the rotational speed of the mechanical equipment and blade excited vibration frequency on the blade deformation, the authors analyze the data of the experimental results. And according to the Fourier transform spectrum of the frequency domain of the test signal, it is found that the reasons of blade deformation are mainly composed of two parts: the rotation of the mechanical equipment of the low frequency band and the vibration of the high frequency band. In this experiment, the mechanical speed is lower than $1800 \mathrm{rpm}$, which means the frequency is lower than $30 \mathrm{~Hz}$. The selected blade of the stimulated natural frequency is about $250 \mathrm{~Hz}$, and the interference frequency in the middle band of $100 \mathrm{~Hz}-200 \mathrm{~Hz}$ range are less (shown in Fig. 4). So in the time domain signal for sub-band processing, the authors choose $120 \mathrm{~Hz}$ as the frequency dividing point, and we hold that the frequency range $0 \mathrm{~Hz}-$ $120 \mathrm{~Hz}$ of the signal is caused by the rotating speed of the mechanical equipment; and when the frequency of the signal is greater than $120 \mathrm{~Hz}$, the blade excited vibration is caused by uncertain factors such as unstable mechanical system, air resistance, and other factors.

Figures 5 and 6 , respectively, show that mechanical speed and fiber grating wavelength changes in the relationship between the two figures with the crack depths of $h=0.2 \mathrm{~mm}$ and $h=0.5 \mathrm{~mm}$. It can be seen that when the speed of rotation of the mechanical equipment increases, the total wavelength variation of the fiber grating and the change in the wavelength of the fiber grating caused by the excited vibration of the blade also show an 
increasing trend. But the wavelength of the fiber grating caused by the rotation of the device does not increase, presenting a horizontal trend. The reason may be that the shape of the blade is relatively simple, and the size is small. In the $2000 \mathrm{rpm}$ speed range, the effect of air resistance on the blade is not enough obvious, and the blade has been in the laboratory environment in the idle running state, so the speed of the blade deformation is small. However, the effect of stimulated self-vibration on

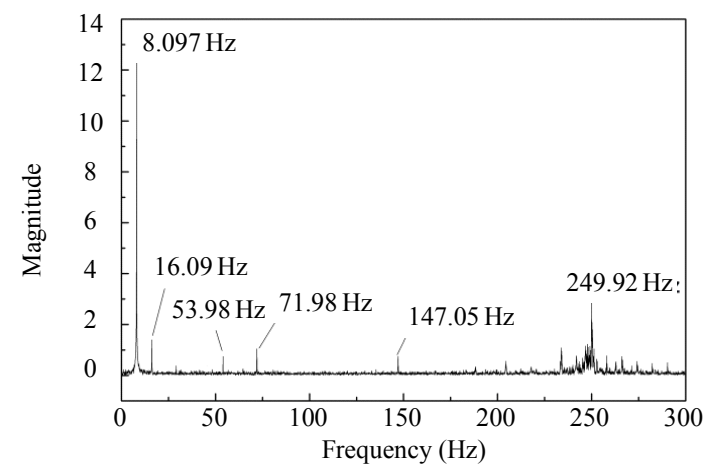

(a) the blade deformation is mainly due to the instability of the whole rotating system. The installation of the equipment causes the rotor to be unbalanced. As the speed increases, the vibration of the mechanical system becomes more and more obvious, as well as the presence of various frequency multiplication signals. And the intersection of the signal phase leads to the vibration, which is caused by the larger deformation of the blade.

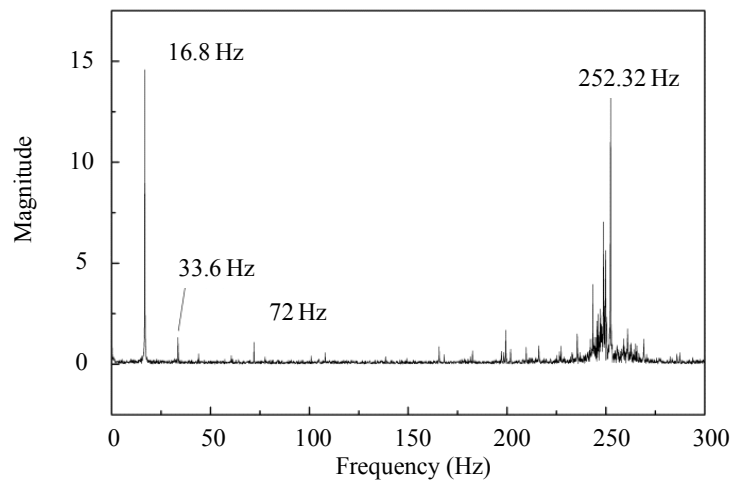

(b)

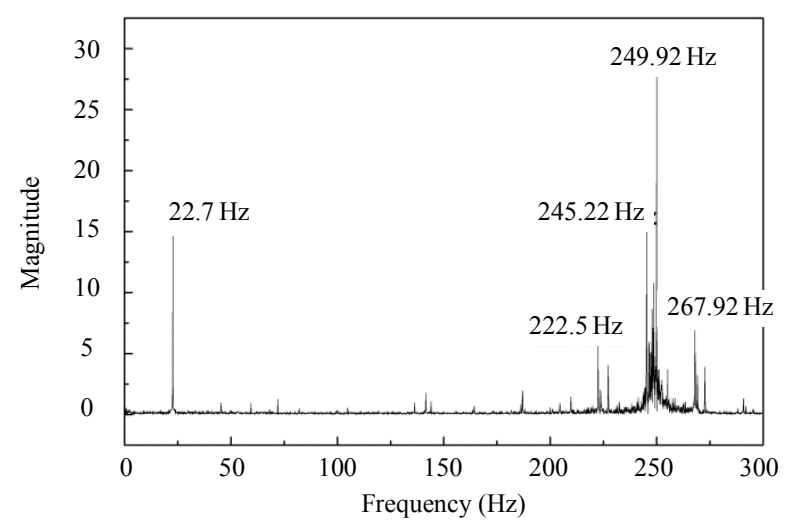

(c)

Fig. 4 Spectrum in different speeds: (a) $v=485 \mathrm{rpm}$, (b) $v=1005 \mathrm{rpm}$, and (c) $v=1355 \mathrm{rpm}$.

A careful study of the experimental results shows that the total value of the wavelength variation of the fiber grating is slightly larger than the change in the grating wavelength caused by the self-vibration of the blade, and both of these values are proportional to the rotational speed. In the case of a crack depth $h=0.2 \mathrm{~mm}$, the wavelength variable grows slowly at a relatively low speed stage $(v<1000 \mathrm{rpm})$. When the rotational speed is greater than $1000 \mathrm{rpm}$, the wavelength increases with an increase in the speed; when the rotational speed is less than $800 \mathrm{rpm}$, the wavelength variation caused by the speed is greater than the wavelength variation caused by blade self-vibration. When the rotation speed is higher than $800 \mathrm{rpm}$, the wavelength variable caused by the stimulated self-vibration of the leaf plays a leading role in the total wavelength variable. In the whole speed range, the wavelength 
variation caused by the speed basically remains stable, which is always about $8 \mathrm{pm}$. And when the speed reaches $1500 \mathrm{rpm}$, the wavelength variation caused by the blade excited vibration is more than $25 \mathrm{pm}$, and the wavelength of the total value is greater than $30 \mathrm{pm}$. We will find that the total value of the wavelength change is not simply a sum of the simple geometries equal to the self-magnitude and the speed value, because there is a phase problem between the signals in each band.

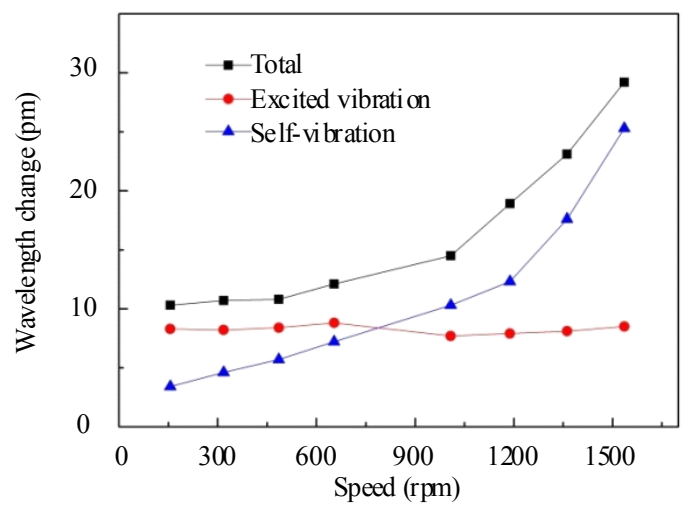

Fig. 5 Relationship between the rotational speed and wavelength of the fiber grating $(h=0.2 \mathrm{~mm})$.

Figure 6 shows the results of the blade when the crack depth $h$ is $0.5 \mathrm{~mm}$, which is similar to that of the crack depth $h=0.2 \mathrm{~mm}$, and has a consistent curve trend. The difference is that when the rotational speed reaches $600 \mathrm{rpm}$, the wavelength variation caused by the blade excited vibration has become greater than that caused by the rotary speed of mechanical device.

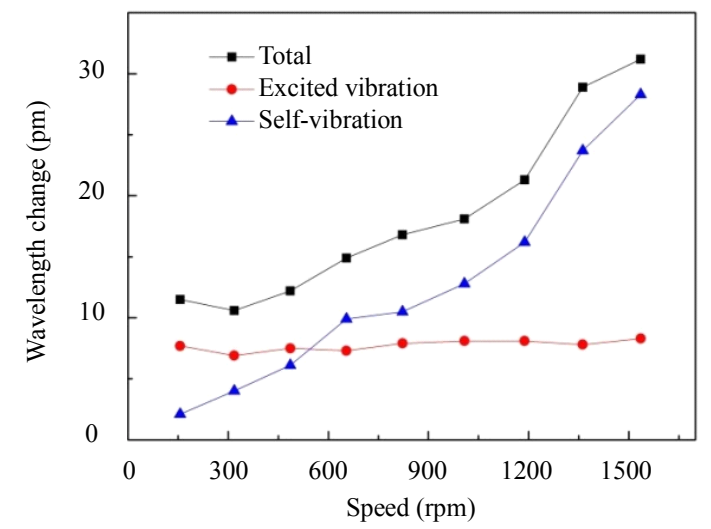

Fig. 6 Relationship between the rotational speed and wavelength of the fiber grating $(h=0.5 \mathrm{~mm})$.
Figure 7 summarizes the total value of the fiber grating wavelength variation at different speeds with different depths of through-cracked blades, as well as the magnitude of the error between multiple experiments and long-term data collection. It can be clearly seen from the figure that at the same speed, there is a clear relationship between the change in the wavelength and the depth of the crack, which means the larger the crack depth is, the longer the wavelength of the fiber grating is. And the greater the crack depth is, the longer the wavelength is as the speed increases, and the greater the test error value is. When the rotational speed is $1500 \mathrm{rpm}$, the total wavelength variation of the complete blade is less than $20 \mathrm{pm}$, and the total length of the blade with the crack depth of $h=0.2 \mathrm{~mm}$ is about $25 \mathrm{pm}$, whose test error is no more than $5 \mathrm{pm}$. However, when $h=0.9 \mathrm{~mm}$, the test error is more than $45 \mathrm{pm}$, whose error is also reached as much as $10 \mathrm{pm}$. And when the speed is less than $600 \mathrm{rpm}$, all the different conditions of the leaves of the test results are less than $20 \mathrm{pm}$, whose test error is also smaller.

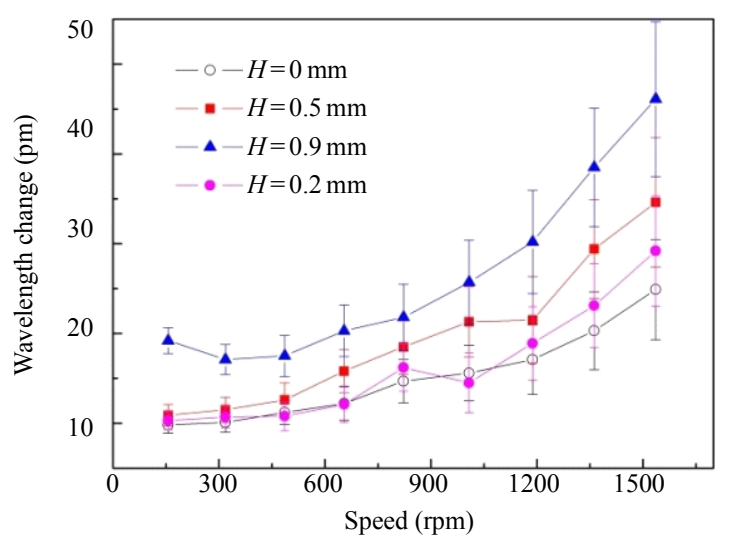

Fig. 7 Relationship between the blade deformation and speed.

In order to verify the correctness of the above analysis and find out the better judgment method for the running state detection of the blade, the authors further deal and summarize the blade excited vibration mentioned in the preceding section. The relationship between the natural frequency and the mechanical speed of the different crack depth blades is shown in Fig. 8. It can be seen from the figure that 
the natural frequency of the blade increases with an increase in the mechanical rotary speed. The changes in the natural frequencies of the complete and smaller crack blades are more consistent with the change in the rotary speed. Besides, the natural frequency of the blade is the largest, and the vibration frequency of the blade decreases with an increase in the crack depth of the blade. And from the spectrum (as shown in Fig.4), we can find that in low speed the spectrum is "clean", with less interference signals. And in the scope of $0 \mathrm{~Hz}-$ $100 \mathrm{~Hz}$, only a few frequencies and their frequency signals exist. And only a vibration signal in the small range of the natural frequency of the blade exists over $200 \mathrm{~Hz}$ band, and the signal intensity of the natural frequency of the blade is much lower than that of the frequency signal, which is also consistent with the analysis result of the previous wavelength. At high rotational speeds, the signal in the range of more than $200 \mathrm{~Hz}$ is gradually increased, and the signal strength is significantly enhanced. There is little dissonance. A careful observation reveals that many signals are in the form of a frequency band centered at the natural frequency.

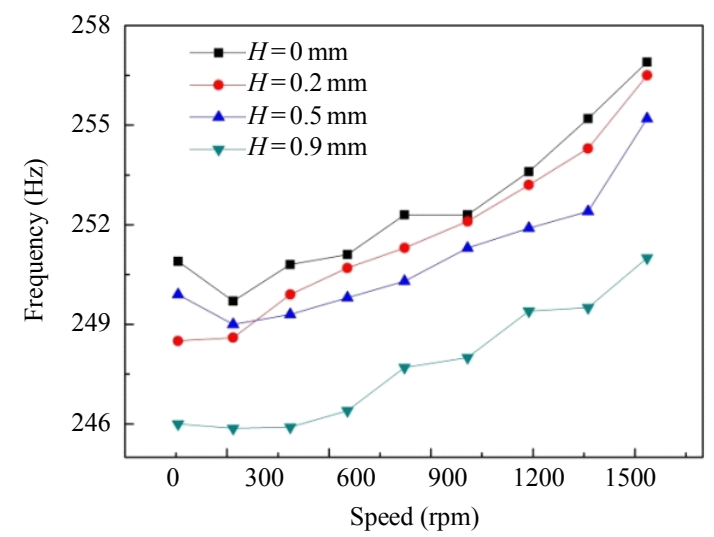

Fig. 8 Relationship between the blade natural frequency and speed.

The analysis of many phenomena shown in Figs. 7 and 8 can be explained by the fact that the instability of the mechanical rotation system causes the disturbance of the natural frequency of the blade. Higher mechanical speed can provide higher stiffness and increase its natural frequency of the blade; the depth of the crack acts in the opposite direction to the mechanical speed, reducing the stiffness of the blade, resulting in a decrease in the natural frequency, and more irregular frequency signals appear.

\section{Conclusions}

(1) The deformation of the blade is mainly composed of two parts: the speed of the mechanical equipment and the self-vibration of the blade. The influence of the fundamental frequency on the deformation is relatively constant within the experimental speed range; the main effect is stemmed from the natural frequency of the blade. The higher the rotational speed is, the greater the effect of self-vibration on blade deformation is.

(2) An increase in the crack depth will not only increase the deformation of the blade, but also reduce the natural frequency of the blade.

\section{Acknowledgement}

This work was supported in part by the Project of Key Consulting Research of Chinese Academy of Engineering (2016-XZ-13), Project of the Key Laboratory for Health Monitoring and Control of Large Structures (201505), Science and technology planning project of Zhongshan City, Guangdong Province (2015A2028), and Project of Hubei Engineering University (Nos: 201513 and 201611).

Open Access This article is distributed under the terms of the Creative Commons Attribution 4.0 International License (http://creativecommons.org/ licenses/by/4.0/), which permits unrestricted use, distribution, and reproduction in any medium, provided you give appropriate credit to the original author(s) and the source, provide a link to the Creative Commons license, and indicate if changes were made.

\section{References}

[1] Y. D. Wang, L. Q. Zhu, H. M. Shi, and B. Q. Guo, "Research on real-time vision detection technology for motion feature of high-speed mechanical system," Journal of Mechanical Engineering, 2016, 52(2): 82-90. 
[2] Q. B. Tong, H. P. Ma, L. H. Liu, X. D. Zhang, and G. B. Li, "Key technology study on radial vibration detection system of high-speed rotating machinery," Chinese Journal of Scientific Instrument, 2011, 32(5): 1026-1032.

[3] G. M. J. Herbert, S. Iniyan, E. Sreevalsan, and S. Rajapandian, "A review of wind energy technologies," Renewable and Sustainable Energy Reviews, 2005, 11(6): 1117-1145.

[4] Q. G. Chen and C. Wang, "Advances in research and application of vibration monitoring technology for rotating blades based on blade tip timing method," Noise and Vibration Control, 2016, 36(1): 1-5.

[5] R. Tomassini, G. Rossi, and J. F. Brouckaert, "On the development of a magnetoresistive sensor for blade tip timing and blade tip clearance measurement systems," AIP Conference Proceedings, 2014, 1600:
$65-73$.

[6] Z. D. Zhou, Y. G. Tan, M. Y. Liu, W. Yang, and Z. Y. $\mathrm{Li}$, "Actualities and development on dynamic monitoring and diagnosis with distributed fiber Bragg grating in mechanical systems," Journal of Mechanical Engineering, 2013, 49(19): 55-69.

[7] Y. J. Wang, L. Liang, Y. Q. Yuan, G. Xu, and F. Liu, "A two fiber Bragg gratings sensing system to monitor the torque of rotating shaft," Sensors, 2016, 16(1): 138-1-138-8.

[8] S. Kuhnt, J. Wemicke, J. Shadden, and R. Byars, "Advanced wind turbine controls input based on real time loads measured with fibre optical sensors embedded in rotor blades," The European Wind Energy Conference \& Exhibition, 2006, 2: 1224-1230. 